\title{
Modeling of COVID-19 Transmission under Markov Chains in Uganda
}

\author{
Raphael Naryongo ${ }^{1,{ }^{*}}$, Joab Onyango ${ }^{2}$, Loyford Njagi $^{2}$, Margaret Nakirya ${ }^{3}$ \\ ${ }^{1}$ Department of Statistics and Actuarial Science, Jomo Kenyatta University of Agriculture and Technology, Nairobi, Kenya. \\ ${ }^{2}$ Department of Mathematics, Meru University of Science and Technology, Meru, Kenya. \\ ${ }^{3}$ Department of Mathematics, Kampala International University, Kampala, Uganda.
}

How to cite this paper: Raphael Naryongo, Joab Onyango, Loyford Njagi, Margaret Nakirya. (2022) Modeling of COVID-19 Transmission under Markov Chains in Uganda. Journal of Applied Mathematics and Computation, 6(1), 4-12.

DOI: 10.26855/jamc.2022.03.002

Received: November 24, 2021

Accepted: December 17, 2021

Published: January 4, 2022

*Corresponding author: Raphael Naryongo, Department of Statistics and Actuarial Science, Jomo Kenyatta University of Agriculture and Technology, Nairobi, Kenya.

Email:

raphael.naryongo@students.jkuat.ac.ke

\begin{abstract}
In this research study, we have modelled the transmission of COVID-19 in Uganda using a discrete-time Markov chain. Most of the already used epidemiological or infectious disease transmission models consist of partial differential equations that do not generalize the determinants of transition at discrete-time intervals when estimating the probability transition matrix. However, using the historical data provided by the Ugandan government through daily press statements, the model has revealed the state of transmission within the population. Furthermore, our model had shown that it is easier to deal with the disease at a latency stage than when the transmission had grown explosively among the healthy Ugandans. In addition, the findings of the research study should enable the Ugandan government to take appropriate preventive disease control measures when combating this life-threatening global pandemic.
\end{abstract}

\section{Keywords}

Discrete-time Markov Chains, Transmissions, Probability Transition Matrix, HCDR Model, COVID-19 Pandemic

\section{Introduction}

Since the inception of COVID-19 in December last year, several epidemiological and deterministic mathematical models have been proposed to model the disease transmission dynamics to help the world understand the transmission of the virus from one state to another during a specified period. In addition, there has been turmoil worldwide, especially with the higher number of deaths experienced globally [1]. The COVID-19 is a novel coronavirus transmitted instantaneously among humans and was discovered in China in December 2019. The disease has spread now to almost 99 percent of all countries or over 300 countries worldwide. COVID-19 is a severe respiratory virus transmitted through several avenues via contact with an infected person. As of November 10th, 2021, over two hundred million people were confirmed with the virus; simultaneously, more than five million people succumbed due to the virus globally.

The transmission of the COVID-19 virus occurs primarily through close contact with a person with the confirmed virus. This is by tiny droplets produced when the confirmed person coughs, sneezes, or talks. Research by [2] proved that the virus is not airborne, meaning that the spread cannot be via the air over long distances. However, many models mainly apply to non-stochastic dynamics in nature, which cannot ascertain the virus metrics on the probability of infection or recovery on the first patient and the expected time of disease for those COVID-19 persons (either healthy or have the virus).

A person in a healthy state may also contract the COVID-19 virus by touching a contaminated surface while touching their face. The virus may survive on surfaces for up to three days or approximately seventy-two hours in most cases. The 
virus is most contagious in its first three days after symptoms, commonly known as the symptomatic stage. The disease can also be transmitted without showing immediate signs through an asymptomatic stage. A person may transmit the disease to others without showing it at the initial stages, only to show symptoms at latency stages, according to [3]. The number of days a person takes before showing the signs of COVID-19 may vary from one individual to another according to their levels of immunity, which may start from three to 14 days maximum.

The most common symptoms that a person can exhibit include high fever, dry cough, and in some cases, shortness of breath. The common forms of body complications for a confirmed incident may consist of mild to acute pneumonia, and many other respiratory distress syndromes may occur, whether mild or sharp. For now, the primary treatment is symptomatic and mechanisms of supportive therapy by [4]. However, putting in place preventive measures like wearing musk, frequent handwashing with flowing water, or using hand sanitizers, covering of mouth when one is coughing, among other measures, can help reduce the spread of the virus.

Many countries have put in more efforts to prevent the spread of the COVID-19 virus, which includes restrictions on traveling as in Uganda, quarantines for the confirmed cases, curfews for healthy persons. Other measures are workplace hazard controls, cancellations of planned events, social facility closures, and event postponements, especially in the sporting arena. The pandemic has now led to unblemished disruption of global socioeconomic according to [5] and ([6], which has a massive impact on people's livelihoods. This has led to a shortage supply caused by panic buying at the same time in countries. Almost 95 percent of schools have been affected, leaving a potential economic recession in over 205 countries globally.

For instance, [7] formulated a mathematical model, which is discrete when investigating the transmission of SARS. The findings noted that early and strict quarantine measures were vital for SARS control. [8] and [9] did develop ordinary differential equations and stochastic SEIR models when studying the infectious disease dynamics before proposing the most effective control measures in terms of interventions [10]. While the models have been used in several Ebola pandemic outbreaks in many African countries such as DRC, it is essential to note that new models that use probability are more precise in transmission control of the COVID-19 models. Many models have been used in East Africa to model the transmission of COVID-19 such stochastic modeling, as well as prediction of the COVID-19 pandemic and economic effects, spread in Kenya [11] and [12].

This research study uses a discrete-time Markov chain model for estimating the transmission rates of COVID-19 in Uganda. One can model the Coronavirus transmission discretely to help policymakers implement the best strategies that can work well for control measures, thus preventing unwanted deaths. The deaths have been poor modeling techniques from other commonly used epidemiological models of disease transmission in Uganda. The COVID-19 will always affect the business activities in many countries in terms of the stock securities market in the world, such as the Nairobi Securities Exchange market [13] and general economic performance. We have structured this research paper as follows; introduction and literature review in Chapter 1, methodology in Section 2, sources of data used during modeling in Section 3, data analysis, and interpretation of results in section four.

\section{Methodology}

\subsection{Modeling Preliminary Techniques}

\subsubsection{HCDR Conceptual Framework}

In this research study, it is important to consider four discrete states, namely, Healthy (state 0), Corona-ill (state 1), Recovered (state 2) and Dead (state 3) denoted with acronym as HCDR. These states can be represented in a conceptual/methodological framework as follows:

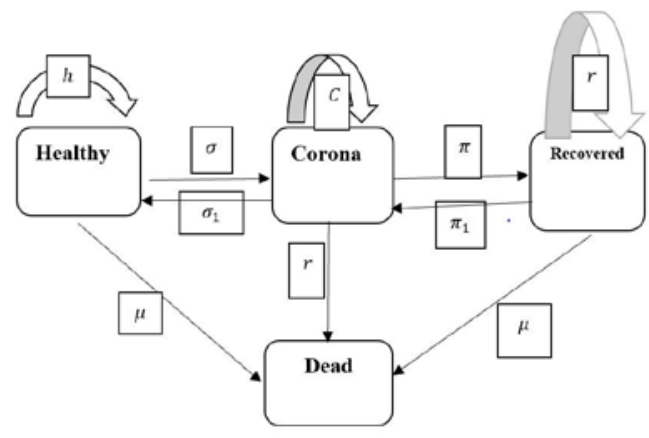

Figure 1. Conceptual Framework of COVID-19 Transmission. 
From Figure 1, the states can be defined as discrete-time states. Let $\left(Y_{i}, i=0,1,2,3\right)$ to represent number of persons who are at any given state from COVID-19 in Uganda at time, $t$. This means that the stochastic process, $Y_{t}$ is a stochastic process that has states namely $0,1,2$ and 3 . Thus, the 1st-order dependency in terms of time-homogeneous

Definition 1. Markov chain used to model the process statistically is Yet defined as follows;

$$
P\left[Y_{n+1}=y / Y_{1}=y_{1}, Y_{2}=y_{2}, Y_{3}=y_{3} \ldots \ldots . . Y_{n}=y_{n}\right]=P\left[Y_{n+1} 1=y / Y_{n}=y_{n}\right]
$$

provided that the initial conditions are defined as follows;

$$
P\left[Y_{1}=y_{1}, Y_{2}=y_{2} \ldots, Y n=y_{n}\right]>0
$$

From equation 1 and 2, the Markov chain will be;

$$
P\left[Y_{n}=i_{n} / Y_{n}-1=i_{n}-1\right]
$$

Thus, the transition probability matrix defined as

$$
P_{i, j}=\left[\begin{array}{llll}
p_{00} & p_{01} & p_{02} & p_{03} \\
p_{10} & p_{11} & p_{12} & p_{13} \\
p_{20} & p_{21} & p_{22} & p_{23} \\
p_{30} & p_{31} & p_{32} & p_{33}
\end{array}\right]
$$

where we can define, $\sum_{j=0}^{3} P_{i j}=1$ provided that states $i$ can take the values, $i=0,1,2,3$.

\subsubsection{Definitions of COVID-19 States of Nature}

From the (HCDR) Conceptual Framework of COVID-19 Transmission in Figure 1, we will define the states as follows;

- Healthy State $(H)$ : This comprises of those persons who have not been exposed to COVID-19.

- $\quad$ Corona State $(C)$ : This comprises of persons who have been confirmed with COVID-19 virus in Uganda.

- $\quad$ Dead State $(D)$ : This comprises of persons who have died from the COVID-19 in Uganda.

- $\quad$ Recovered $(R)$ : It comprises of those who have recovered from the Corona virus.

The probability transition matrix, $P_{i, j}$ denotes the probability of moving from state $i$ to state $j$ within a single time period. For instance, $p_{00}$ is defined as the probability that a healthy person (state 0 ) will still be healthy (state 0 ) from time 0 to time 1 and $p_{23}$ is the probability that a person who was in state 2 will be in state 3 after a given time period, $t=1$.

Based on the assumptions of the above model, the next state of a person only depends on the previous state as from equation (3). When a person stays in a state where he or she cannot be removed by the existing modes of decrements such as healthy or dead state, this is called an absorbing state. The transition probabilities in the transition probability matrix remain constant during the period of transmission.

\subsection{Probability Transition Matrix Model}

In this model formulation, we will use the maximum likelihood estimation to estimate of the probability transition matrix for the four states of the COVID-19 transmission. The four states are as in Table 1.

Table 1. COVID-19 Transmission States in Uganda

\begin{tabular}{ccccc}
\hline Groups & Healthy & Corona & Recovery & Dead \\
\hline Healthy & $Y 00$ & $Y 01$ & $Y 02$ & $Y 03$ \\
Corona & $Y 10$ & $Y 11$ & $Y 12$ & $Y 13$ \\
Recovery & $Y 20$ & $Y 21$ & $Y 22$ & $Y 23$ \\
Dead & $Y 30$ & $Y 31$ & $Y 32$ & $Y 33$ \\
\hline
\end{tabular}

From Table 1: $Y_{00}$ : number of Healthy persons exposed to COVID-19 in Uganda but still remains healthy by the time study ends; $Y_{01}$ : number of persons who contracted the COVID-19 virus by the time study ended; $Y_{02}$ : number of persons who contracted the virus but recovered at the end of the study; $Y_{03}$ : the number of people who died at the end of study period. In addition, $Y_{10}$ : number of persons who recovered from COVID-19 at the end of the period of study; $Y_{11}$ : number of infected persons who remained infected by the time the study ends; $Y_{12}$ : number of persons who recovered from the virus by the time study ends; $Y_{13}$ : the persons who died by the time study ended. $Y_{20}$ : the people who recovered from the virus and become healthy again, $Y_{21}$ : those who contracted the virus after recovery; $Y_{22}$ : those who contracted the virus but remained with the virus by the time study ended and $Y_{23}$ : the number of people who died from COVID-19 after recovery from the virus. In the end, $Y_{30}$ : number of people who died from exposed but not related to COVID-19; $Y_{31}$ : 
people who died from the virus after contracting the virus: $Y_{33}$ : number of people who died after recovery from the virus; $Y_{33}$ : number of people who died from the virus.

The number of dead people in $Y_{30}, Y_{31}, Y_{32}$ would be zero because death is an absorbing state. In addition, the probability of staying in the state until the end of the study is one such as $Y_{33}=1$. The vector matrix for the $\left(Y_{30}, Y_{31}, Y_{32}, Y_{33}\right)$ would be $(0,0,0,1)$.

Definition 2. The property of independence of the Markov chains is assumed in this study, the stated likelihood of the probability transitions, $P_{i, j}$, is modeled as a binomial model as follows;

$$
L\left(P_{i, j} / \boldsymbol{\theta}, y\right)=\left(\begin{array}{c}
\boldsymbol{\theta}_{i} \\
y_{i, j}
\end{array}\right) P_{i, j}^{y_{i, j}}\left(1-P_{i, j}\right)^{\boldsymbol{\theta}_{i}-y_{i, j}}
$$

where the $\theta_{i, j}$ is the observed number of transitions that starts from states $i$ to $j$ during the period of study and the probabilities are constant during the entire period of study assumption is made. The $P_{i, j}$ is estimated from the multi-binomial estimation method from equation (5) as;

$$
\hat{P_{i, j}}=\frac{y_{i, j}}{\sum_{\substack{\forall j \\ y_{i, j}}}}=\frac{y_{i, j}}{\boldsymbol{\theta}_{i}}
$$

for the values of $i, j=0,1,2,3$, the standard errors have been estimated from the data given by the Ugandan government on the Covid-19 pandemic as a stated sampling distribution is;

$$
\hat{s} . e\left(\hat{P_{i, j}}\right)=\sqrt{\frac{\hat{P_{i, j}}\left(1-\hat{P_{i, j}}\right)}{\theta_{i}}}
$$

\subsection{Estimating Covid-19 Metrics}

In estimating metrics of the pandemic, we assume that probability that a healthy person will be confirmed with Covid-19 for the first time between time $t-1$ and $t$ steps for the states of nature, $i, j=0,1$ as from the probability transition matrix described above is;

$$
\begin{gathered}
g_{0,1}^{(t)}=P\left[Y_{x+t}=1, Y_{x+t-1}=0, \ldots Y_{x+1}=0 / Y_{x}=0\right] \\
g_{0,1,2}^{(t)}=p_{00}^{t-1} p_{00}
\end{gathered}
$$

From the same argument in equation (8), the number of Covid-19 confirmed persons who recover between time $t-1$ and $t$ steps will be;

$$
\begin{gathered}
g_{1,0}^{(t)}=P\left[Y_{x+t}=0, Y_{x+t-1}=1, \ldots Y_{x+1}=1 / Y_{x}=1\right] \\
g^{1,1}=p_{11}^{t-1} p_{10}
\end{gathered}
$$

Ultimately, the expected number of pandemic confirmed cases and recoveries will be having a close form solution that can be computed as;

$$
\begin{gathered}
E\left[\Theta_{i, j}^{1}\right]=\frac{\sum_{t=1}^{\infty} t * g_{i, j}^{t}}{P(i \longrightarrow j)} \\
E\left[\Theta_{i, j}^{1}\right]=\frac{1}{1-p_{i, i}}
\end{gathered}
$$

For values of $i, j=0,1$ and $\mathrm{i}$ is not equal to $\mathrm{j}$, where $\sum_{t=1}^{\infty} t * g^{t}{ }_{i, j}$ is defined as mean passage time from one state $\mathrm{i}$ to another state $\mathrm{j}$ while $\mathrm{P}(\mathrm{i} \rightarrow \mathrm{j})$ is defined as $E\left[\theta^{1}{ }_{i, j}\right]=\frac{1}{1-p_{i, j}} *\left(-p_{i, j}\right)=\frac{p_{i, j}}{1-p_{i, j}}$. This is defined as the ultimate probability of moving from one state $\mathrm{i}$ to state $\mathrm{j}$. The number of days a person confirmed with corona virus lives from the state to another state $H_{i}$ where $i=0,1$ is estimated using the equation.

Where I is a 2 by 2 matrix denoted as $P=\left\{\begin{array}{ll}p_{00} & p_{01} \\ p_{10} & p_{11}\end{array}\right\}$

$$
H=(I-P)^{-1}\left(\begin{array}{l}
1 \\
1
\end{array}\right)
$$

\subsection{Steady State Analysis}

At the equilibrium state, the method of estimating the steady state analysis of the transition probabilities was proposed by [16]. The $P_{i, j}^{X}, i, j=0,1,2,3$, which are the COVID-19 states are called the probability transition matrix and is computed using a decomposition method that requires the eigenvalues and their equivalent eigenvectors. Thus, it can be estimated as 


$$
P^{x}=W \Lambda^{x} W^{-1}
$$

where $W$ is a 4 by 4 matrix with values $Y_{0}, Y_{1}, Y_{2}, Y_{3}$ and $Y_{j}$, for $j=0,1,2,3$ are the right Eigen-vectors that are equivalent to the eigen-values $\pi_{j}$, for $j=0,1,2,3$. Therefore,

$$
\begin{gathered}
P Y_{j}=\pi_{j} Y_{j} \\
\Lambda^{x}=\left\{\begin{array}{cccc}
\pi_{0}^{x} & 0 & 0 & 0 \\
0 & \pi_{1}^{x} & 0 & 0 \\
0 & 0 & \pi_{2}^{x} & 0 \\
0 & 0 & 0 & \pi_{3}^{x}
\end{array}\right\}
\end{gathered}
$$

\section{Source of Covid-19 Data}

The Ugandan government provided all data used in this study through the Ministry of Health website database. The data is from 13th May 2020 until 6th September 2021, when this study was ready for submission. The data used in this research is available at the Ministry of Health of Uganda and the World Health Organization Websites, respectively. One has to register online before can be able to get access the data.

The analysis was done using statistical programming languages called $\mathrm{R}$ and Excel Spread Sheet. The results are discussed in the next section 4 as well as the graphical representations.

From the data from the Ministry of Health in Uganda, the data are given as follows; Healthy/healthy=200,986, ill/ill=10,652, recovered/ill=3,570, healthy/dead=92, ill/healthy=10,840, ill/ill=1,001, ill/recovered=998, ill/dead=41, recovered/healthy=2,418, recovered/ill=802, recovered/re recovered/dead=22 and all deaths were 471 . The above information shows the number of persons per state by the end of the study as at 7th September 2021.

\section{Data Analysis}

\subsection{The Conceptual Framework Model}

We have assumed that the discrete-time states of the applied Markov chain model for COVID-19 pandemic as healthy at (state 0 ), confirmed with the virus at (state 1), recovered from the virus at (state 2) and dead at (state 3 ) states. Also, Let $Y_{i}$, for $i=0,1,2,3$ to denote the number of persons who are at any given state from COVID-19 at time, $t$. The discrete-time Markov chain model will have a state space, $S\{i\}$ for $i=\{0,1,2,3\}$.

\subsection{Estimation of the Transition Probabilities for COVID-19 Pandemic}

The maximum likelihood method was used during estimation of the transition probability matrix for the COVID-19 pandemic. Table 2 shows the expected estimates as well as levels of confidence intervals for the Corona virus transition probabilities respectively.

Table 2. Maximum Likelihood Estimates of COVID-19 transition probabilities

\begin{tabular}{cccc}
\hline Estimated Parameters & $P^{\wedge} i, j$ & $\hat{s}^{\wedge} e\left(P_{i, j}^{\wedge}\right)$ & $95 \%$ confidence intervals \\
\hline$p 00$ & 0.850 & 0.00283 & $0.84505-0.85305$ \\
$p 01$ & 0.090 & 0.00263 & $0.08950-0.09060$ \\
$p 02$ & 0.000 & 0.0000 & $0.0000-0.0000$ \\
$p 03$ & 0.001 & 0.00973 & $0.00099-0.00102$ \\
$p 10$ & 0.810 & 0.00972 & $0.80940-0.81020$ \\
$p 11$ & 0.100 & 0.00785 & $0.09915-0.10250$ \\
$p 12$ & 0.080 & 0.00753 & $0.07945-0.00825$ \\
$p 13$ & 0.010 & 0.00186 & $0.00985-0.01020$ \\
$p 20$ & 0.810 & 0.00789 & $0.80850-0.810350$ \\
$p 21$ & 0.100 & 0.00872 & $0.09955-0.10180$ \\
$p 22$ & 0.080 & 0.00723 & $0.07985-0.00835$ \\
$p 23$ & 0.010 & 0.00541 & $0.00995-0.01021$ \\
$p 30$ & 0.000 & 0.00000 & $0.00000-0.00000$ \\
$p 31$ & 0.000 & 0.00000 & $0.00000-0.00000$ \\
$p 32$ & 0.000 & 0.00000 & $0.00000-0.00000$ \\
$p 33$ & 1.000 & 1.00000 & $1.00000-1.00000$ \\
\hline
\end{tabular}


The transition probability matrix is then determined as;

$$
P_{\text {Covid }-19}=\left[\begin{array}{ccccc}
* & 0 & 1 & 2 & 3 \\
0 & 0.850 & 0.090 & 0.059 & 0.001 \\
1 & 0.810 & 0.100 & 0.080 & 0.010 \\
2 & 0.810 & 0.100 & 0.080 & 0.010 \\
3 & 0.000 & 0.000 & 0.000 & 1.000
\end{array}\right]
$$

A graphical algorithm can sometimes be used when representing the transition probabilities matrix for COVID-19, thus enabling an easy classification of the states into transient, recurrent, or an absorbing state. Equation 15 shows how the COVID-19 has been classified into different states. In addition, state $\{0\}$, state $\{1\}$ and state $\{2\}$ are both transient and recurrent states while state $\{3\}$ is known as an absorbing state.

For example, state $\{0,1\}$ is said to be transient since the two states can communicate with one another at the same time; it has a negative probability ever of going back to the given class. State $\{3\}$ is called an absorbing state for the COVID-19 virus since once a person is dead, he or she stays dead with a probability of one (which is a certainty probability). The model of discrete-time Markov chain cannot be regarded as ergodic even if it is aperiodic. It is because of its irreducible property for all the available states that don’t belong to a similar equivalence class.

\subsection{Estimation of COVID-19 Metrics}

\subsubsection{The 1st Transition Probability of COVID-19 Pandemic}

The probability of a healthy person being confirmed with the COVID-19 case in Uganda was determined by equation (8). Moreover, the probability that a Coronavirus confirmed person will become healthy or recovers has been estimated using equation (9). Figure 2 shows the distribution of the transition probabilities of the virus across several discrete-time steps from point one to a hundred. The figure also shows how the disease slowed down on the confirmed statistics of the infected persons. This phenomenon may be due to the high level of education that the Ugandan government has taken into consideration by ensuring that the population gets more information on how to combat the spread of the COVID-19 virus.

In addition, when a person is confirmed with the virus at an early, initial, or latent stage, the government needs to improve higher rates of recovery while reducing the chances of infecting the healthy population. The results also show a small probability of recovering from the virus disease after it has been moved to several future discrete time steps.

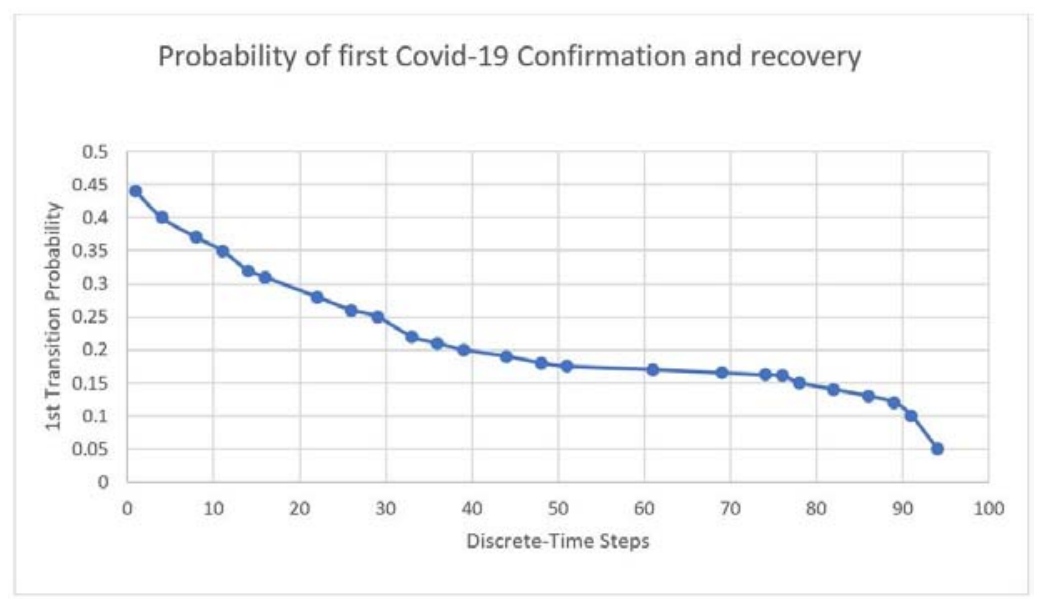

Figure 2. Probability of First COVID-19 Confirmation and Recovery.

\subsubsection{The Probability of COVID-19 Pandemic Transmissions}

At any time, the probability of COVID-19 transmission at any time, $t$, was determined using the cumulative totals of the probabilities of 1st confirmed cases of healthy persons. Figure 3 shows a graph of the cumulative transition probabilities from the matrix over discrete-time intervals. From the diagram, it is easy to note that the virus has become more pandemic over time as the study progresses within the healthy population. This phenomenon is similar to what happened in the USA after the healthy population ignored the government and WHO measures [7] to stay home and avoid spreading the virus. The disease transmission has become more exponential, thus prompting desperate curative measures such as country lock-down to prevent further spread. 


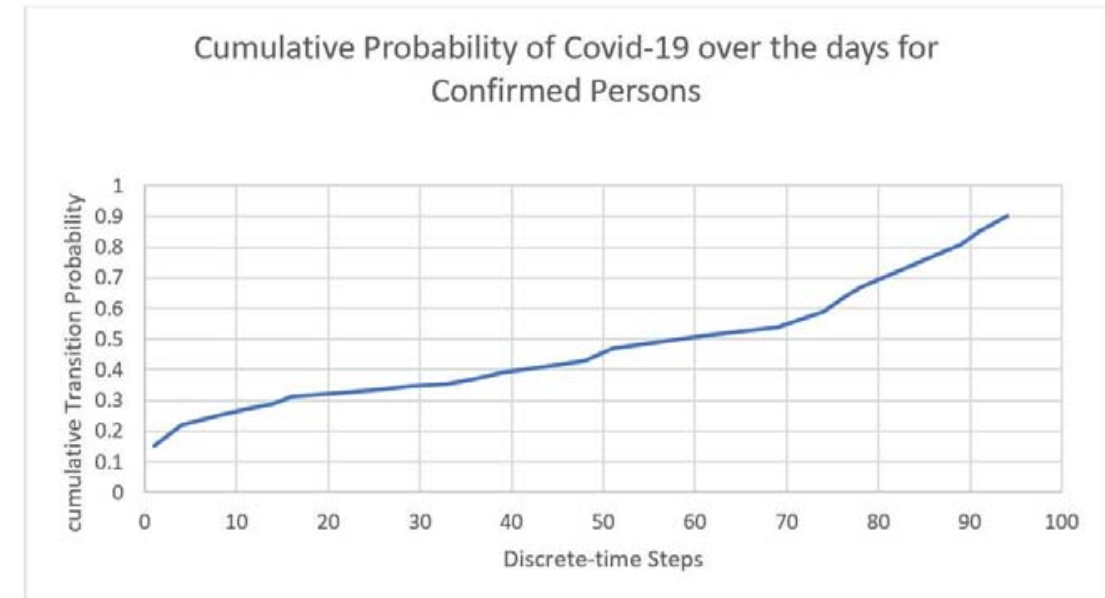

Figure 3. Cumulative Probability of COVID-19 over the days for Confirmed Persons.

\subsection{COVID-19 Pandemic Expected Confirmation \& Recovery Time}

The expected time for confirmation and recovery was determined using equation (10) for the coronavirus. The expected time for proof of a healthy person was 10.25 days in a given cohort of persons considered in the study. The average time for a healthy person to be exposed to the virus was between 2 and 4 days. Time to recovery is a summation given that the virus takes 5 to 14 days before it becomes symptomatic; otherwise, it is just asymptomatic in the other states.

While using this discrete-time Markov chain model, the number of deaths of persons who have been confirmed with the virus remains at a rate of 8 percent, which is far above the WHO's mortality rate of 2 to 3 percent. This spike in cases is from the recklessness of Ugandans to the virus's exposure, especially when they do not follow the COVID-19 containment guidelines.

Table 3. Estimates of COVID-19 Metrics in Uganda

\begin{tabular}{cc}
\hline Metrics & COVID-19 Pandemic \\
\hline General likelihood of confirmation/Infection & 0.85 \\
General likelihood of Recovery & 0.92 \\
Expected time to Confirmation (in days) & 12 days \\
Expected time to Recovery (in days) & 32 days \\
Probability of Reinfection & 0.50 \\
\hline
\end{tabular}

From Table 3, it is essential to note that the general likelihood of a person living in Uganda contracting the COVID-19 virus is $85 \%$ at the same time, the recovery rate is 92 percent. It means that the death rate is $8 \%$, which is still high compared to the most affected countries globally, such as the USA, with $7.5 \%$ of all the people who have contracted the virus. The expected number of days until the confirmation of the virus is 12 days, which is slightly below the maximum number of days for all symptoms to show up in 14 days. This phenomenon may be attributed to the tropical conditions that are being experienced in Uganda.

The recovery period is 32 days, which includes the 14 days for Quarantine. It is defined as the amount of time it takes for a healthy person who has just contracted the COVID-19 virus to recover. However, there is the risk of reinfection when the person exposes himself or herself to the virus again. The probability of reinfection from the COVID-19 in Uganda stands at $50 \%$. It means that half of those healthy persons who have recovered from the virus did not develop a more robust immune system to withstand another virus's case if they get into contact again.

The Ugandan government needs to ensure that the Coronavirus survivors do not get reinfected following the exposure again from the contaminated area to prevent the spread among millions of healthy persons within the period.

\subsection{Estimation of the Equilibrium State Transition Probability Matrix}

The $P^{x}$ as $x^{-} \rightarrow \infty$, from definition of [15] which is the transition probability matrix had been estimated from equation (12) that showed that the discrete-time Markov chain is not irreducible but aperiodic since the removed state (3), death is 
an absorbing state. This can help in predicting the transition probability matrix for transition probabilities for the Covid-19 at any given time, $t$-step. This means that the Covid-19 probability transition matrix at equilibrium state will be;

$$
P_{\text {Covid-19 }}^{x}=\left[\begin{array}{ccccc}
* & 0 & 1 & 2 & 3 \\
0 & 0.850^{x} & 0.090^{x} & 0.059^{x} & {\left[1-\left(0.850^{x}+0.090^{x}+0.059^{x}\right)\right.} \\
1 & 0.810^{x} & 0.100^{x} & 0.080^{x} & {\left[1-\left(0.810^{x}+0.100^{x}+0.080^{x}\right)\right.} \\
2 & 0.810^{x} & 0.100^{x} & 0.080^{x} & {\left[1-\left(0.810^{x}+0.100^{x}+0.080^{x}\right)\right.} \\
3 & 0 & 0 & 0 & 1
\end{array}\right]
$$

Clearly when the value of $x=1$, then we have equation (16), from the determined $P_{\text {Covid }}{ }^{1}-19$ transition matrix that gives the actual first transition matrix for the corona virus. Henceforth, whenever the value of $x \geq 2$, then transition matrix can be easily generated from the modeled $P_{\text {Covid }}{ }^{x}-19$ matrix even as $x$ becomes larger. However, it is important to assume that the transition probabilities are constant over the duration of the period of study.

From equation (16), a researcher or reader of the paper can obtain the probabilities of the different states of a healthy person who have the pandemic virus from time, $x=2,3$, 4,. until where $x$ is a large number (as $x-\rightarrow \infty$ ) commonly known as in the long run probabilities of the transition matrix operations.

\section{Conclusion}

From the above scientific research, the discrete-time Markov chain model has revealed that COVID-19 has a favorable transmission rate initially. It becomes more severe in the higher confirmed cases when the healthy persons interact with the confirmed victims. The confirmed cases can grow exponentially to reach a similar situation to that in the USA or even death rates comparable to those in the USA. Therefore, the Uganda government needs to ensure and emphasize the appropriate use of the preventive measures available before focusing on curative measures, especially for the healthy population.

The study findings should help the Ugandan government take necessary precautions on how to deal with COVID-19. There are chances of having a second wave of the pandemic, which is likely to be experienced in the country just like other countries in Europe and Asia. As a recommendation, the discrete-time Markov chain model is thus an excellent epidemiological modeling technique for estimating transmission trends of infectious diseases such as the COVID-19 pandemic.

\section{References}

[1] Mradul Kumar Daga, et al. "From SARS-CoV to Coronavirus Disease 2019 (COVID-19)-A Brief Review”. In: Journal of Advanced Research in Medicine (E-ISSN: 2349-7181 \& P-ISSN: 2394-7047) 6.4 (2019), pp. 1-9.

[2] Ensheng Dong, Hongru Du, and Lauren Gardner. (2020). "An interactive web-based dashboard to track COVID-19 in real time”. In: The Lancet infectious diseases (2020).

[3] Stephen A. Lauer, et al. (2020). "The incubation period of coronavirus disease 2019 (COVID-19) from publicly reported confirmed cases: estimation and application”. In: Annals of internal medicine (2020).

[4] Jianjun Gao, Zhenxue Tian, and Xu Yang. (2020). "Breakthrough: Chloroquine phosphate has shown apparent efficacy in treatment of COVID-19 associated pneumonia in clinical studies”. In: Bioscience trends (2020).

[5] Warwick J. McKibbin and Roshen Fernando. (2020). "The global macroeconomic impacts of COVID-19: Seven scenarios”. In: (2020).

[6] Joab Odhiambo, Patrick Weke, and Philip Ngare. (2021). “A Deep Learning Integrated Cairns-Blake-Dowd (CBD) Sytematic Mortality Risk Model”. In: Journal of Risk and Financial Management 14.6 (2021), p. 259.

[7] Yicang Zhou, Zhien Ma, and Fred Brauer. (2004). "A discrete epidemic model for SARS transmission and control in China”. In: Mathematical and Computer Modelling 40.13 (2004), pp. 1491-1506.

[8] Phenyo E. Lekone and Barbel F. Finkenstadt. (2006). "Statistical inference in a stochastic epidemic SEIR model with control intervention: Ebola as a case study”. In: Biometrics 62.4 (2006), pp. 1170-1177.

[9] Gerardo Chowell, et al. (2004). "Model parameters and outbreak control for SARS”. In: Emerging Infectious Diseases 10.7 (2004), pp. 1258-9.

[10] Odhiambo, J. O., Ngare, P., Weke, P., and Otieno, R. O. (2020). Modelling of Covid-19 transmission in kenya using 
compound poisson regression model. Journal of Advances in Mathematics and Computer Science, 101-111.

[11] Joab Onyango Odhiambo, Jacob Oketch Okungu, and Christine Gacheri Mutuura. (2020). "Stochastic Modeling and Prediction of the COVID-19 Spread in Kenya”. In: Engineering Mathematics (2020).

[12] Joab Odhiambo, Patrick Weke, and Philip Ngare. (2020). "Modeling Kenyan economic impact of corona virus in Kenya using discrete-time Markov chains”. In: Journal of Finance and Economics 8.2 (2020), pp. 80-85.

[13] Joab Odhiambo, Patrick Weke, and Jusper Wendo. (2020). "Modeling of Returns of Nairobi Securities Exchange 20 Share Index Using Log-Normal Distribution”. In: (2020).

[14] Chih-Cheng Lai, et al. (2020). "Severe acute respiratory syndrome coronavirus 2 (SARSCoV-2) and corona virus disease-2019 (COVID-19): the epidemic and the challenges”. In: International Journal of Antimicrobial Agents (2020), p. 105924.

[15] Ahmed Merie, Myron Hlynka, et al. (2019). "Medical Intervention for Disease Stages Using Game Theory, Markov Chains, and Bayesian Inference”. In: International Journal of Statistics and Probability 8.4 (2019), pp. 60-67.

[16] U. Narayan Bhat and Gregory K. Miller. (2002). Elements of applied stochastic processes. Vol. 3. Wiley-Interscience Hoboken^eN. JNJ, 2002. 